\title{
Sulla geometria differenziale degli spazi vettoriali.
}

\author{
Nota di E. Bompiani (a Roma) \\ Alla memoria di Guido Castelnuovo, nel primo centenario della nascita.
}

Sunto. - Costruzioni geometriche di invarianti differenziali (di linee o di ipersuperificie) in uno spazio centro-affine.

1. - In un eccellente volumetto uscito da poco, D. LAUgWITZ prende a considerare la geometria differenziale degli spazi vettoriali (anche nel caso, e anzi con particolare enfasi, di infinite dimensioni) come la geometria di uno spazio centro-affine $\left({ }^{1}\right)$.

Come l'A. osserva, la geometria centro-affine attuale è cominciata con ricerche di E. R. LORCH $\left({ }^{2}\right)$ negli spazi di BANAOH (e non negli spazi di dimensione finita).

Il Ladgrta comincia a definire in uno spazio centro-affine di dimensione finita una metrica riemanniana, che è quella del LoroH nel caso in esame, e per mezzo di essa un elemento d'arco, un vettore normale ed una curvatura di una curva (piana) in un punto.

Gli invarianti non son quelli introdotti da E. SALKowsKI ( $\left.{ }^{3}\right)$ e il LaUgWITZ mostra il vantaggio dei nuovi invarianti rispetto a quelli già noti.

In questa nota riprendo la trattazione per indicare ulteriori costruzioni e proprietà relative sia ad elementi di ipersuperficie che di curve in uno spazio centro-affine.

\section{2. - Elemento lineare determinato da un $E^{2}$.}

In un piano centro-affine assumiamo il centro $O$ come origine di coor. dinate affini $x, y$.

Data una curva $C$ e un suo punto regolare (non di flesso) $A \neq O$ si può scegliere la retta $O A$ come asse $y$ e il punto $A$ come $(0,1)$ e l'asse $x$ paral. lelo alla tangente in $A$ alla curva $C$.

Una conica $\Gamma$ è fissata dall' $E^{2}$ della curva in $A$ (ciò̀ dall'avere ivi contatto del $2^{\circ}$ ordine con $C$ ) e dall'avere $O$ per centro di simmetria.

Per le scelte già fatte questa conica ha un'equazione del tipo

$$
y^{2}-1=h x^{2}
$$

(1) D. LaUGWraz, Differentialgeometrie in Vehtorïumen unter besonderer Beriucksichti. gung der unendlichen Räume; Vieweg \& Sohn, Braunschweig.

(2) E. R. Lorok, A curvature study of convex bodies in Banach spaces; Ann. di Mate. matica, s. IV, t. XXXIV, 1953, p. $105=112$.

(3) E. Salkowski, Affine Differentialgeometrie; Berlin-Leipzig 1934. 
e si fissa il punto unità sull'asse $x(e$ con cio si determina tutto il riferimento) prendendo $h= \pm 1$ (uguale $a-1$ se $\Gamma$ è un'ellisse, $a+1$ se è un'iperbole).

Per un elemento $E^{1}$ di componenti $d x, d y$ spiccato dal punto $A$ si può introdurre un invariante infinitesimo legato ad una forma quadratica. Sulla retta parallela per $O$ all'elemento dato si consideri l'elemento e una intersezione della retta con $\Gamma$ : il rapporto semplice dei tre punti cosi definiti sulla retta è in valore assoluto

$$
d s=\left|\sqrt{d y^{2}-h \overline{d x^{2}}}\right| .
$$

Si può prendere questa come «lunghezza » del vettore infinitesimo di com. ponenti $d x, d y$; essa è determinata da un $E^{2}$.

$\grave{\mathbf{E}}$ pare determinata da questo una direzione, quella della retta congiun. gente i punti-unità sugli assi, che però non prende il mome di direzione normale ad $E^{2}$.

\section{3. - Curvatura d'un $E^{3}$ piano.}

Passiamo ad esaminare un $E^{3}$ nel piano centro-affine. Supporremo che la conica $\Gamma$ (per l' $E^{2} \subset E^{3}$ e simmetrica rispetto ad $O$ ) sia un'ellisse (cose analoghe si trovano se è un'iperbole) e che l' $E^{3}$ sia rappresentato da

$$
y-1=-\frac{1}{2} x^{2}+c x^{3}+[4]
$$

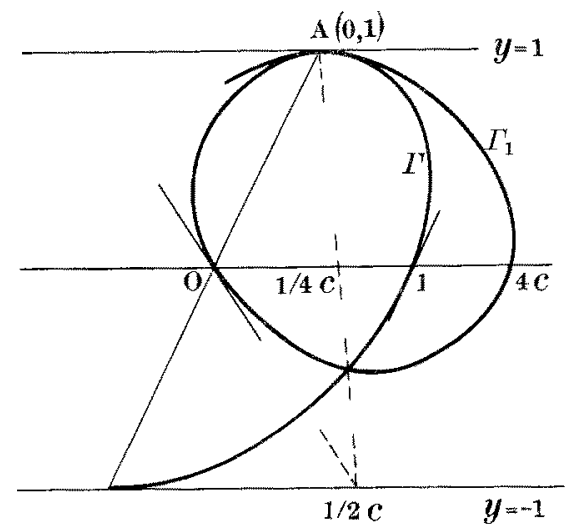

Si trova senza difficoltà che la conica $\Gamma_{1}$ contenente l' $E^{3}$ e passamte per $O$ ha l'equazione

$$
2(y-1)(y+2 c x)+x^{2}=0 .
$$


Il punto $\neq O$ in cui questa conica $\Gamma_{1}$ taglia l'asse $x$, di coordinate $(4 c, 0)$ caratterizza geometricamente $c$.

Poichè il punto unità sull'asse $x$ era definito (come intersezione con $\Gamma$ ) a meno di una simmetria ora possiamo individuarlo in modo che risulti $c>0$ se non è $c=0$.

Anche la tangente in $O$ a $\Gamma_{1}, y+2 c x=0$, caratterizza $c$; e così la retta $4 c x+y-1=0$, che congiunge $(0,1)$ all'ulteriore punto d'intersezione di $\Gamma \in \Gamma_{1}$.

Queste due rette s'incontrano nel punto $(1 / 2 c,-1)$. Gli elementi trovati permettono facilmente di tracciare $\Gamma_{1}$ (per $c \neq 0$ ). L' $E^{3}$ (per l' $E^{2}$ dato) per cui $c=0$ si caratterizza anche facilmente. $L^{\prime} E^{3}: y-1=-\frac{1}{2} x^{2}+[4]$ e il suo simmetrico rispetto ad $0: y+1=\frac{1}{2} x^{3}+[4]$ appartengono alla stessa conica $\left(x^{2}+y^{2}=1\right)$ individuata da $E^{2}$ e dal suo simmetrico rispetto ad 0 ; ciò̀ è l' $E^{3}$ in $A$ di questa conica. Questo è il significato geometrico di $c=0$.

Se si vogliono mettere l'invariante infinitesimo $d s$ e quello finito (c) ora trovato in relazione con l'elemento d'arco e la curvatura del LAUGWITz, basta ricordare anzitutto che se $x(t), y(t)$ sono coordinate affini su una curva del piano, qualunque sia il parametro $t$, deve aversi

$$
\frac{d^{2} x}{d t^{2}}+a(t) \frac{d x}{d t}+b(t) x=0, \frac{d^{2} y}{d t^{2}}+a(t) \frac{d y}{d t}+b(t) y=0
$$

e che si può scegliere un parametro $s(t)$ tale che

$$
\frac{d^{2} x}{d s^{2}}+a(s) \frac{d x}{d s}+x=0, \frac{d^{2} y}{d s^{2}}+a(s) \frac{d y}{d s}+y=0
$$

(queste equivalgono all'equazione vettoriale (17) a p. 10 del LAUGwITZ; $d s$ è l'elemento d'arco affine).

Riferendoci all' $E^{3}(3.1)$ si ha per $x=0$ :

$$
y=1, \frac{d y}{d x}=0, \frac{d^{2} y}{d x^{2}}=-1, \frac{d^{3} y}{d x^{3}}=\sigma c, \frac{d x}{d s}=1, \frac{d y}{d s}=0
$$

e dalle equazioni differenziali, sempre per $x=0$ è

$$
\frac{d^{2} x}{d s^{2}}=-a(0), \frac{d^{2} y}{d s^{2}}=-1
$$

Per derivazione dall'equazione differenziale in $y(s)$

$$
\frac{d^{3} y}{d s^{3}}=-\frac{d y}{d s}\left\{1+\frac{d a(s)}{d s}\right\}+a(s)\left\{y(s)+a(s) \frac{d y}{d s}\right\}
$$


e per $x=0$

$$
\frac{d^{3} y}{d s^{3}}=a(s)
$$

D'altra parte

$$
\frac{d^{3} y}{d s^{3}}=\frac{d^{3} y}{d x^{3}}\left(\frac{d x}{d s}\right)^{3}+3 \frac{d^{2} y}{d x^{2}} \frac{d^{2} x}{d s^{2}} \frac{d x}{d s}+\frac{d y}{d x} \frac{d^{3} x}{d s^{3}}
$$

e per $s=x=0$ in base alle determinazioni precedenti

ciò̀

$$
a(0) \sigma=c+3 a(0)
$$

$$
a(0)=-3 c .
$$

Questa relazione e la costruzione precedente danno il significato geometrico della curvatura affine (e della normale affine).

L'osservazione già fatta che un $E^{3}$ per cui $c=0$ (quindi $a=0$ ) appartiene alla conica con centro in $O$ determinata dal suo $E^{2}$ porta senz'altro al teorema di LAUGWITz per cui le uniche curve a curvatura affine nulla sono le coniche con centro in $O$.

\section{4. - Quadriche osculatrici.}

In uno spazio centro-affine $A_{n+1}$ consideriamo una calotta $n$-dimensionale del $3^{0}$ ordine $\sigma_{n}^{3}$. Se $O$ è il centro di $A_{n+1}$ e $A$ il centro della $\sigma_{n}^{3}$ possiamo sempre scegliere coordinate affini $x^{i}(i=1, \ldots, n)$ e $z$ tutte nulle in $O$, tali che in $A$ siano $x^{i}=0, z=1$, e finalmente che l'iperpiano tangente in $A \alpha \sigma_{n}^{3}$ sia $z=1$. La rappresentazione di $\sigma_{n}^{3}$ è del tipo

$$
z-1=\varphi_{2}(x)+\varphi_{3}(x)+[4]
$$

ove $\varphi_{s}$ è una forma di grado $s$ nelle $x^{i}$ e [4] indica termini arbitrari d'ordine $\geq 4$ nelle $x^{i}$.

Una quadrica simmetrica rispetto ad $O$ passante per $A$ con iperpiano tangente $z=1$ ha un'equazione del tipo

$$
z^{2}-1=\Phi_{2}(x)+z \Phi_{1}(x)
$$

con $\Phi_{\sigma}$ forma di grado $s$ nelle $x^{i}$.

Essa contiene $\sigma_{n}^{2}$ appartenente a $\sigma_{n}^{3}$ se e solo se $\Phi_{2}(x)=2 \varphi_{2}(x)$ cioè la quadrica centrale (di centro $O$ ) osculatrice è la

$$
Q_{0}: z^{2}-1=2 \varphi_{2}(x)
$$


La $Q_{0} \supset \sigma_{n}^{3}$ se e solo se è $\varphi_{3}(x) \equiv 0$; se ciò avviene in ogni punto di una ipersuperficie questa è una quadrica con centro in $O$; e questo equivale al teorema di LaUGwitz espresso dall'equazione (12) a pag. 27.

Proouriamoci ora l'equazione di una quadrica per $\sigma_{n}^{3}$ (che diremo ancora osculatrice) con centro nel punto $\left(a^{i}, z_{0}\right), z_{0} \neq 1$.

Una quadrica $Q$ con centro in questo punto ha equazione del tipo

$$
Q: \alpha_{i k}\left(x^{i}-a^{i}\right)\left(x^{k}-a^{k}\right)+2 \alpha_{i}\left(x^{i}-a^{i}\right)\left(z-z_{0}\right)+\alpha\left(z-z_{0}\right)^{2}-1=0 .
$$

Se $\sigma_{n}^{2}$ ̀े rappresentata da

$$
z=1+\varphi_{2}(x)+[3]=1+\varphi_{i k} x^{i} x^{k}+[3]
$$

affinchè la quadrica $Q \supset \sigma_{n}^{2}$ bisogna che sostituita questa espressione di $z$ nella sua equazione risultino identicamente nulli i termini di grado 0, 1, 2 nelle $x^{i}$. Ciò dà luogo alle condizioni seguenti:

$$
\begin{aligned}
& \alpha_{i k} a^{i} \dot{a}^{k}-2 \alpha_{h} a^{h}\left(1-z_{0}\right)+\alpha\left(1-z_{0}\right)^{2}=1 \\
& \alpha_{i k} a^{k}=\alpha_{i}\left(1-z_{0}\right) \\
& \alpha_{i k}-2 \alpha_{h} a^{h} \varphi_{i k}+2 \alpha\left(1-z_{0}\right) \varphi_{i k}=0 .
\end{aligned}
$$

Dalle ultime due si può ricavare $\alpha_{i k} a^{i} a^{k}$ ed eliminando nei dne modi possibili quest'espressione fra le tre equazioni che la contengono si ha

di cui

$$
\begin{aligned}
& \alpha_{h} \alpha^{h}\left(1-z_{0}\right)-\alpha\left(1-z_{0}\right)^{2}=-1 \\
& \alpha_{h} \alpha^{h}\left\{2 \varphi_{2}(a)-1+z_{0}\right\}-2 \alpha_{\varphi_{2}}(\alpha)=0
\end{aligned}
$$

$$
A=\alpha_{h} a^{h}=-\frac{2 \varphi_{2}(a)}{\left(1-z_{0}\right)^{2}}, \alpha=-\frac{2 \varphi_{2}(a)-\left(1-z_{0}\right)}{\left(1-z_{0}\right)^{3}}
$$

$e$ in conseguenza delle equazioni date

$$
\alpha_{i k}=-\frac{2 \varphi_{i k}}{1-z_{0}}, \alpha_{i}=-\frac{2 \varphi_{i k} a^{k}}{\left(1-z_{0}\right)^{2}}
$$

Il fatto che le $\alpha_{i k}$ non dipendano dalle $a^{i}$, ma solo da $z_{0}$ prova il teorema di L.Adgwitz secondo il quale due quadriche osculatrici $\supset \sigma_{n}^{2}$ aventi i centri sopra un medesimo iperpiano parallelo a quello tangente a $\sigma$ sono segate da tale jperpiano in due quadriche (a $n-1$ dimensioni) che si ottengono l'una all'altra per traslazione (quella che porta un centro nell'altro). 
Si può osservare che la quadrica osculatrice di centro $\left(a^{i}, z_{0}\right)$ contiene il punto improprio della retta $O A$ se $\alpha=0$ cioè se

$$
1-z_{0}=2 \varphi_{2}(a)
$$

cioè se il centro appartiene alla quadrica

$$
1-z=2 \varphi_{2}(x)
$$

Questa si caratterizza rispetto alla quadrica osculatrice centrale (con centro o) $Q_{0}$ col fatto che $Q$ contiene le sezioni di $Q_{0}$ con $z=1$ e $z=-2$ e il punto improprio di $O A$. Perciò :

Le $\infty^{n}$ quadriche osculatrici passanti per il punto improprio di OA sono quelle aventi $i$ centri sulla quadrica $Q$ che ha in comune con $Q_{0}$ le sezioni prodolle dai piani $z=1$ e $z=-2$ e contenente lo stesso punto improprio.

\section{5. - Calotte del $3^{\circ}$ ordine e quadriche osculatrici.}

Passiamo ora a considerare una calotta del $3^{\circ}$ ordine $\sigma_{n}^{3}$ (di equazione 4.1) e le quadriche ad essa osculatrici, cioè contenenti la $\sigma_{n}^{2}$.

L'intersezione della quadrica $Q$ (di centro $a^{i}, z_{0}$ ) con $\sigma_{n}^{3}$ ha in $A$ (centro della calotta: $\left.a^{i}=0, z=1\right)$ un punto triplo e le tangenti in esso appartengno all'iperpiano $z=1$ e in esso al cono

$$
\alpha_{i} x^{i} \varphi_{2}(x)-A \varphi_{3}(x)+\alpha\left(1-z_{0}\right) \varphi_{3}(x)=0
$$

e per le espressioni già trovate di $\alpha_{i}, A, \alpha$

$$
\varphi_{3}(x)=2 \varphi_{i k} x^{i} \dot{a}^{k} \varphi_{2}(x)
$$

Questa equazione dipende dalle $a^{i}$ ma non da $z_{0}$; ciò mostra che:

Tutte le quadriche osculatrici aventi $i$ centri su una parallela alla retta $O A$ (congiungente il centro dello spazio affine col centro di $\sigma_{n}^{2}$ ) danno luogo ad intersezioni con $\sigma_{n}^{3}$ aventi lo stesso cono cubico tangente.

Possiamo chiederci se è possibile che il cono delle tangenti asintotiche di $\sigma_{n}^{2}$ e il cono cubico ora trovato riescano apolari. Perchè ciò avvenga, posto

$$
\varphi_{3}(x) \equiv \varphi_{i k} x^{i} x^{k} x^{l}
$$


e indicato con $\varphi^{i k}$ l'elemento reciproco di $\varphi_{i k}$ in $\operatorname{Det}\left(\varphi_{i k}\right) \neq 0$ deve aversi

cioè

$$
\varphi_{i k l} \varphi^{k l}=2 a^{h} \varphi_{h}\left(i \varphi_{k l}\right) \varphi^{k l}
$$

$$
\varphi_{i k l \varphi^{k l}}=\frac{2}{3}(n+2) \varphi_{i h} a^{h}
$$

o più esplicitamente

$$
a^{h}=\frac{3}{2(n+2)} \varphi^{h i} \varphi_{i k l} \varphi^{k l}
$$

Poichè $\approx_{0}$ rimane arbitrario, si ha che:

Se $\varphi_{2}(x)$ è una forma non-degenere esistono $\infty^{1}$ quadriche osculatrici, $i$ cui centri $\left(a^{h}, z_{0}\right)$ stanno sopra una ben determinata retta parallela ad OA, tali che il loro cono quadrico nell'iperpiano tangente in $A$ e il cono cubico prima trovato siano apolari.

\section{6. - Elementi differenziali curvilinei.}

Abbiamo iniziato con lo studio di un elemento curvilineo $E^{2}$ o $E^{3}$ in un piano centro-affine e siamo poi passati allo studio di calotte d'ipersuperficie in uno spazio centro-affine $A_{n+1}$.

Occupiamoci ora di elementi $E^{n+2}$ in $A_{n+1}$.

Serviamoci di coordinate affini $x, y^{2}, y^{3}, \ldots y^{n}, z$ tali che nel centro $O$ dello spazio affine siano $x=y^{i}=z=0(i=2, \ldots, n)$; nel centro $A$ di $E^{n+1}$ siano $x=y^{i}=0, z=1$ e $y^{s}=y^{s+1}=\ldots=y^{n}=0, z=1$ con $s \geq 2$ le equazioni dello spazio $S_{s-1}$ oseulatore in $A$ ad $E^{n+1}$ e $z=1$ quella dell'iperpiano osculatore.

Presa $x$ come variabile indipendente la $y^{i}$ ha uno sviluppo in $x$ che comincia col termine in $x^{i}$ ( $i$ è qui esponente) e $z-1$ con $x^{n}$.

Ora è chiaro che con sole combinazioni lineari (cioè sostituendo ad ogni coordinata una conveniente combinazione lineare di essa e delle seguenti) si possono prendere le coordinate in modo che su $E^{n+1}$

$$
y^{i}=\alpha^{i} x^{i}+[n+2], i=2, \ldots, n
$$

$(i \dot{e}$ un indice ordinale in $y \mathrm{e}$ in $\alpha$, e invece un esponente in $x) \mathrm{e}$

$$
z-1=\alpha x^{n+1}+[n+2]
$$

Se $\alpha>0$ sỉ può fare $x=h x^{\prime}$ in modo che $\alpha h^{n+1}=1$ e se chiamiamo $x$ la nuova $x^{\prime}$ l'ultima equazione si scrive

$$
z-1=\alpha^{n+1}+[n+2]
$$


e le precedenti conservano lo stesso aspetto (con diversi valori per i coefficienti $\alpha^{i}$ ).

Se $\alpha<0$ con la stessa sostituzione si può fare $\alpha h^{n+1}=-1$ e quindi analogamente a sopra fare

$$
z-1=-x^{n+x}+[n+2]
$$

E poi sostituendo alle $y^{i}$ le $y^{i} / \alpha^{i}$ e indicando di nuovo queste con $y^{i}$ si ha la forma canonica

$$
y^{i}=x^{i}+[n+2], z-1=\varepsilon x^{n+1}+[n+2]
$$

con $i=2, \ldots, n+1$ e $2= \pm 1$ (se $n+1$ ̀े dispari si può far sempre $\varepsilon=+1$; non così se $n+1$ è pari rimanendo nel campo delle trasformazioni reali).

Cerchiamo le trasformazioni affini che lascino fissi $O$ ed $A$ e non alterino la forma canonica. Intanto apparisce subito che deve essere

$$
\begin{aligned}
& z^{\prime}=z, y^{\prime n}=y^{n}, y^{\prime i}=y^{i}+h_{j}^{i} y^{i} \text { con } j>i \\
& x=x^{\prime}+x_{i} y^{i}, \quad i=2, \ldots, n .
\end{aligned}
$$

Poichè le $y^{i}$ devono avere la stessa forma canonica $\left(=x^{i}+[n+2]\right)$ risulta

$$
\begin{gathered}
y^{\prime n}=x^{\prime n}+[n+2]=\left(x^{\prime}+\alpha_{2} x^{\prime 2}+\ldots+\alpha_{n} x^{\prime n}\right)^{n}+[n+2]= \\
=x^{\prime n}+n \alpha_{2} x^{\prime n+1}+[n+2]
\end{gathered}
$$

e da qui si ricava $\alpha_{2}=0$. Poi

$$
\begin{gathered}
y^{\prime n-1}=x^{\prime n-1}+[n+2]=\left(x^{\prime}+\alpha_{3} x^{\prime 3}+\ldots\right)^{n-1}+h_{n}^{n-1} x^{\prime n}+[n+2]= \\
=x^{\prime n+1}+(n-1) \alpha_{3} x^{\prime n+1}+h_{n}^{n-1} x^{\prime n}+[n+2]
\end{gathered}
$$

da cui

$$
\alpha_{3}=h_{n}^{n-1}=0
$$

Analogamente procedendo su

$\underline{u}^{\prime n-2}=x^{\prime n-2}+[n+2]=\left(x^{\prime}+\alpha_{4} x^{\prime 4}+\ldots\right)^{n-2}+h_{n-2}^{n-2} x^{\prime n-1}+h_{n}^{n-2} x^{\prime n}+[n+2]$

si trova

$$
\alpha_{h}=h_{n-1}^{n-2}=h_{n}^{n-2}=0
$$


E così continuando si trova che l'unica trasformazione che conserva la forma canonica trovata dell' $E^{n+1}$ ò l'identitò, o in altri termini :

In uno spazio centro-affine un $E^{n+1} d i$ cui nessuno spazio osculatore (punto, tangente, piano osculatore, ...) sia incidente al centro dello spazio determina un riferimento affine.

Conseguenza immediata di ciò è che un $E^{n+1+s}$ ammette $n s$ invarianti, i quali tuttavia potranno non essere indipendenti (come di certo accade per una curva algebrica se $s$ è abbastanza grande).

\section{7 - Elementi differenziali in $A_{3} e$ in $A_{4}$.}

Limitiamoci a considerare un elemento curvilineo $E^{4}$ in uno spszio $A_{\mathrm{s}}$ centro-affine.

Sia esso rappresentato da

$$
y=x^{2}+c x^{4}+[5], z=1+x^{3}+d x^{4}+[5]
$$

Le quadriche con centro $O$ contenenti $E^{3} \subset E^{4}$ sono le $\infty^{z}$ rappresentate da

$$
z^{2}-1=a_{11}\left(x^{2}-y z\right)+2 x y+a_{22} y^{2}
$$

con $a_{11}$ e $a_{22}$ arbitrarii. Le quadriche contenenti $E^{4}$ formano un fascio determinato dalle due quadriche

$$
x^{2}-y z+c y^{2}=0, z^{2}-1=2 y(x+d y) .
$$

La prima ̀̀ un cono di vertice $O$ che dipende soltanto da $c$, quindi contiene tutti gli $\infty^{x} E^{4}$ che si ottengono al variare di $d$ : questi formano uno psendoelemento $\left({ }^{4}\right)$ rappresentato da

$$
y=x^{2}+c x_{4}+[5], z=1+x^{3}+[4]
$$

(cioè la totalità degli $E^{4}$ per $E^{3}$ appartenenti a questo cono).

La seconda è una quadrica (cui appartiene la tangente in $A$ all'elemento) che dipende solo da $d$, cioè contiene tutti gli $\infty^{1}$ elementi $E^{4}$ che si

${ }^{4}$ Ho introdotto la nozione di pseudoelemento differenziale nella Nota « Nuovi enti gaometrici: pseudoelementi diferenziali ", Rendio Semin. Mat. e Fisico di Milano, vol. XXIII (1963). In essa si trovano numerose citazioni anche in relazione ai pennelli di elementi differenziali e agli elementi composti di cui appresso. 
ottengono al variare di $c$ : questi formano uno pseudoelemento (che è un pennello o elemento composto secondo TERRACINI) rappresentato da

$$
y=x^{2}+[4], z=1+x^{3}+d x^{4}+[5] .
$$

Passiamo a considerare un $E^{5}$ contenente $l^{\prime} E^{4}$ precedente

$$
y=x^{2}+c x^{4}+c^{\prime} x^{5}+[6], z=1+x^{3}+d x^{4}+d^{\prime} x^{5}+[6]
$$

Esso appartiene alla quadrica di centro $O$ :

$$
z\left(d^{\prime}-c\right)\left\{x^{2}-y z+c y^{2}\right\}+\left(c^{\prime}+1\left\{z^{2}-1-2 y(x+d y)\right\}=0\right.
$$

che appartiene, naturalmente, al fascio di quadriche per $E^{4}$.

Per $d^{\prime}=c$ e $c^{\prime} \neq-1$ si ha la quadrica del fascio che ha per generatrice in $A$ la tangente all'elemento $E^{4}$ : questa dipende solo da $d$, ciò ad essa appartengono gli $\infty^{2} E^{5}$ :

$$
y=x^{2}+c x^{4}+c^{\prime} x^{5}+[6], z=1+x^{3}+d x^{4}+c x^{5}+[6]
$$

con $c$ e $c^{\prime}$ arbitrari. Questi $\infty^{3} E^{5}$ costituiscono uno pseudoelemento contenente gli $\infty^{1}$ pennelli

$$
y=x^{2}+c x^{4}+[5], z=1+x^{3}+d x^{4}+c x^{5}+[6]
$$

al variare di $c$.

Se invece è $c^{\prime}=-1$ e $d^{\prime} \neq c$ la quadrica del fascio contenente $E^{5}$ è un cono di vertice $O$ determinato dalla sola $c$, di cui $\grave{\theta}$ generatrice la retta $O A$ e il piano tangente Iungo questa al cono $\otimes$ il piano $y=0$; sicchè apparten. gono a questo cono tutti gli $\infty^{2} E^{5}$ (di centro $A$ )-

$$
y=x^{2}+c x^{4}-x^{5}+[6], z=1+x^{3}+d x^{4}+d^{\prime} x^{5}+[6]
$$

con $d$ e $d^{\prime}$ arbitrari, costituenti ciò̀ lo pseudoelemento

$$
y=x^{2}+c x^{4}-x^{5}+[6], z=1+x^{3}+[4] .
$$

Se infine $c^{\prime}=-1, d=c$ si ha l' $E^{s}$ appartenente alla curva d'interse. zione del fascio di quadriche più volte nominato.

Diamo ancora, per teminare, alcuni risultati relativi ad elementi curvilinei nello spazio $A_{4}$ centro-affine. Un $E^{a}$ rappresentato da

$$
y_{2}=x^{2}+c_{2} x^{5}+[6], y_{3}=x^{3}+c_{3} x^{5}+[6], z=1+x^{4}+c_{4} x^{5}+[6]
$$


(ove le coordinate sono $x, z$ e $y_{2}, y_{3}$ con gli indici in basso invece che in alto come precedentemente) appartiene alle quadriche (tridimensionali)

$$
\begin{aligned}
& x^{2}-y_{2} z+c_{2} y_{2} y_{3}=0, x y_{2}-y_{3} z+c_{3} y_{2} y_{3}=0 \\
& z^{2}=1+2 y_{2}^{2}+2 c_{4} y_{2} y_{3}, x y_{3}=y_{2}^{2}, y_{3}^{2}=0
\end{aligned}
$$

e al sistema lineare $\infty^{4}$ da esse determinato.

Ciascuna delle prime tre quadriche determina uno psendoelemento, contenente $\infty^{2} E^{5}$ (per lo $E^{4}$ che fissa il riferimento) ottenuti fissando il coefficiente che entra nell'equazione della quadrica e lasciando liberi gli altri due coeffieienti $c_{i}$ che figurano nella rappresentazione dell' elemento. $P$. es. quelli appartenenti alla prima quadrica $\left(\supset E^{4}\right)$ determinano lo psendo. elemento

$$
y_{2}=x^{2}+c_{2} x^{5}+[6], y_{3}=x^{3}+[5], z=1+x^{4}+[5] \text {. }
$$

Se si parte da un $E^{6}$ rappresentato da

$$
\begin{aligned}
& y_{2}=x^{2}+c_{2} x^{5}+d_{2} x^{6}+[7], y_{3}=x^{3}+c_{3} x^{5}+d_{3} x^{6}+[7] \\
& z=1+x^{4}+c_{4} x^{5}+d_{4} x^{6}+[7]
\end{aligned}
$$

si trova che le quadriche di centro $O$ per esso sono:

$$
\begin{aligned}
& x^{2}-y_{2} z+c y_{2} y_{3}+\left(d_{2}+1\right) y_{3}^{2}=0, x y_{2}-y_{3} z+c_{3} y_{2} y_{3}+\left(d_{3}-c_{2}\right) y_{3}^{2}=0 \\
& z^{2}-1-2 y_{3}^{2}-2 c_{4} y_{2} y_{3}-2 d_{4} y_{3}^{2}=0, x y_{3}-y_{2}^{2}-c_{3} y_{3}^{2}=0 .
\end{aligned}
$$

Anche qui si possono fare considerazioni analoghe a quelle fatte in casi precedenti. P. es. la prima di queste quadriche contiene $\infty^{4} E^{6}$ per l' $E^{4}$ che determina il riferimento; essi sono quelli che si hanno dalla rappresentazione precedenti lasciando arbitrari $c_{3}, d_{3}, c_{4}, d_{4}$ e costituiscono uno pseudo. elemento che potrà indicarsi con

$$
y_{2}=x^{2}+c_{2} x^{5}+d_{2} x^{6}+[7], y_{3}=x^{3}+[5], z=1+x^{4}+[5] .
$$

L'elemento $E^{6}$ che appartiene alle prime tre quadriche contenenti $E^{5}$ (assegnato, ciò̀ fissate $c_{2}, c_{3}, c_{4}$ ) è caratterizzato da $d_{2}=-1, d_{3}=c_{2}, d_{4}=0$. 